Bundesgesundheitsbl -

Gesundheitsforsch - Gesundheitsschutz

$2000 \cdot 43: 758-769$ ๑ Springer-Verlag 2000

Leitthema: Lebensmittelsicherheit

H. Tschäpe

Robert Koch Institut, Außenstelle Wernigerode

\title{
Lebensmittelbedingte Infektionskrankheiten durch Bakterien
}

\section{Zusammenfassung}

Lebensmittelbedingte Infektionskrankheiten durch Bakterien rufen immer noch eine große öffentliche und gesundheitspolitische Resonanz hervor. Ihre besondere seuchenhygienische Bedeutung ergibt sich daraus, dass sie sich im Lebensmittel rasch vermehren und aus sehr geringen, meist nicht zu entdeckenden Keimzahlen zu klinisch relevanten Zell- oder Toxinkonzentrationen "hoch entwickeln" können. Außerdem verursachen die meisten bakteriellen Erreger wie Salmonellen, Shigellen, Campylobacter, Listerien, EHEC etc. klinisch besonders schwerwiegende Krankheitsbilder. Obwohl entsprechende Ausbrüche meist sehr spektakulär erscheinen, bleiben mit $85 \%$ die sporadischen Fälle von lebensmittelbedingten Infektionskrankheiten epidemiologisch viel wichtiger. Daher müssen die präventiven Maßnahmen auf die Vermeidung der hausgemachten küchenhygienischen Fehler, auf die Reduktion der Erregerlast in den landwirtschaftlichen Tierbeständen sowie auf eine bundesweite Surveillance zur Erfassung überregionaler Ausbrüche gerichtet sein.

\section{Schlüsselwörter}

Lebensmittelverursachte Erkrankungen - Lebensmittelvergiftungen - Gastroenteritiden . Bakterien · Surveillance rotz der großen hygienischen und technologischen Verbesserungen in der letzten Hälfte des 20. Jahrhunderts bei der Herstellung von Lebensmitteln bleiben lebensmittelbedingte Infektionskrankheiten heute immer noch für etwa jeden zehnten Bundesbürger einmal im Jahr ein oft unangenehmes und manchmal klinisch schwerwiegendes Ereignis. Dieses zeigt sich in 95\% der Fälle als Gastroenteritis (Ausnahmen stellen dabei lediglich Infektionen mit Hepatitis A, Listeria monocytogenes, Tuberkulose, Toxoplasmose, etc. dar) und ist als Enteritis infectiosa gemäß Bundes-Seuchengesetz (BSeuchG) meldepflichtig. Solche durch Lebensmittel hervorgerufenen Infektionskrankheiten stehen auf Rang 1 der Häufigkeitsskala übertragbarer Krankheiten in der Bundesrepublik Deutschland und den übrigen europäischen Ländern $[1,2,3]$.

\section{"Durch Lebensmittel hervor- gerufene Infektionskrank- heiten stehen auf Rang 1 der Häufigkeitsskala übertragbarer Krankheiten in der Bundes- republik Deutschland."}

Unter lebensmittelbedingten Infektionskrankheiten - oft auch als mikrobiell bedingte Lebensmittelvergiftungen bezeichnet - versteht man solche Erkrankungen, die nach dem Genuss von Lebensmitteln auftreten und durch Mi- kroorganismen (Viren, Bakterien, Pilze, Parasiten) bzw. durch ihre Toxine bedingt sind, was also auch die bakteriell bedingten Intoxikationen mit einbezieht. Sie begleiten den Menschen seit vielen Jahrhunderten [4] und nehmen in ihrer Häufigkeit und in ihrem Erregerspektrum eher noch zu (vgl. dazu aber weiter unten). Dabei sind die bakteriellen Infektionserreger klinisch und epidemiologisch gesehen von besonderer Bedeutung [5], da sie sich im Lebensmittel z. T. rasch vermehren und relevante Infektionsdosen oder Toxinkonzentrationen erreichen können (vgl. Campylobacter). Auch können bakterielle Gastroenteritiserreger oft zu schweren klinischen Bildern führen, die statistisch häufiger Krankenhauseinweisungen bzw. den Tod zur Folge haben als bei andere Gastroenteritiserregern (Tabelle 1).

Das Spektrum der bakteriellen Lebensmittelvergifter ist sehr vielfältig (Tabelle 2) und ändert sich periodisch gemäß den besonderen sozialen oder seuchenhygienischen Gegebenheiten häufig. Waren vor ca. 100 Jahren lebensmittelbedingte Infektionskrankheiten wie Brucellose, Tuberkulose, Milzbrand, Typhus oder die Tularämie von volksgesundheitlicher Bedeutung, die durch konsequente Ausrottung der Reservoire heute praktisch keine epidemiologische

Prof.Dr.H.Tschäpe

Robert Koch-Institut, Bereich Wernigerode, Burgstraße 37,38855 Wernigerode 


\section{H. Tschäpe}

\section{Foodborne diseases by bacterial pathogens}

\section{Abstract}

Foodborne diseases by bacterial pathogens are of considerable public health concern world-wide. This is supported by their property to propagate in food environments from very small, nearly undetectable numbers to high concentrations of cells or toxins. Moreover, bacterial pathogens such as Salmonellae, Shigellae, Listeriae, or EHEC used to induce serious often also life-threatening illnesses. Although outbreaks of foodborne diseases will always find great public health awareness, sporadic cases remain with $85 \%$ among all foodborne diseases of great epidemiological importance. Therefore, control measures should be directed to establishing a nation wide surveillance system for foodborne infections and to educate peoples with regard to prevention of foodborne diseases.

\section{Keywords}

Foodborne diseases · Food poisoning · Bacteria - Gastroenteritis · surveillance
Rolle mehr spielen [5], so sind es gegenwärtig ganz andere bakterielle Erreger wie Campylobacter, Salmonellen, die Escherichia coli Pathovare, Listerien, die das Infektionsgeschehen beherrschen. Diese bakteriellen Erreger sind dadurch ausgezeichnet, dass sie sich an ihr Reservoir, die landwirtschaftlich genutzten Tierbestände, so ideal angepasst haben, dass sie dort ohne Krankheitssymptome persistieren und so unerkannt und meist nur in geringen Keimzahlen in die Nahrungsmittelkette gelangen können. Man bemerkt sie erst dann, wenn Menschen sich durch kontaminierte Lebensmittel angesteckt haben und wenn spektakuläre Krankheitsausbrüche auftreten. Zudem fördert heute die Globalisierung der Herkunft und der Herstellung der Lebensmittel zusätzlich noch solche Risiken, sich lebensmittelbedingte Infektionskrankheiten zuzuziehen [1].

Es ist daher nicht verwunderlich, dass lebensmittelübertragene Infektionskrankheiten bzw. die damit verbundenen Probleme der Lebensmittelsicherheit gegenwärtig eine große öffentliche Aufmerksamkeit in Presse, Politik und Bevölkerung erlangt haben. Auch die Diskussionen um die Akzeptanz von Novel-Food und die Folgen möglicher BSE-Verseuchung von Rindfleisch und Rindfleischprodukten haben zusätzlich das Bewusstsein der Öffentlichkeit um die Lebensmittelsicherheit verstärkt. Nach dem Motto "Food will keep men healthy but must not turn them ill" (was uns gesund erhalten soll, darf uns nicht krank machen! Robert V. Tauxe auf dem Internationalen Symposium: Salmonella and Salmonellosis, 1997) fragt sich der Verbraucher zunehmend: Warum sind unsere Lebensmittel nicht sicher genug?

\section{"Der Trend zum Verzehr von leicht Gegartem und von rohen Lebensmitteln erleichtert Infektionen über den Lebens- mittelgenuss."}

Der gegenwärtige Trend zum Verzehr von leicht Gegartem und zur Rohkost, insbesondere zu Gemüse (Keimlings-Sprossen), Rohmilch und Rohmilchprodukten, aber auch nach wie vor der Rohfleischverzehr, erleichtern Infektionen über den Lebensmittelgenuss. Auch ermöglichen roheLebensmittelüblicherweise die rasche Vermehrung der Bakterien, die sich dadurch oft nur von verschwindend wenigen Keimen bis zur kritischen Infektionsdosis anreichern können, ohne dass dem Verbraucher das Lebensmittel als auffällig oder gar verdorben auffällt. Viele unserer landwirtschaftlichen Tierbestände sind mit humanpathogenen Durchfallerregern natürlicherweise infiziert, ohne dass man den Tieren dies in ihrem Wohlbefinden anmerkt. Über den Schlacht- und Lebensmittelherstellungsprozess gelangen Spuren der Erreger in die Nahrungsket-

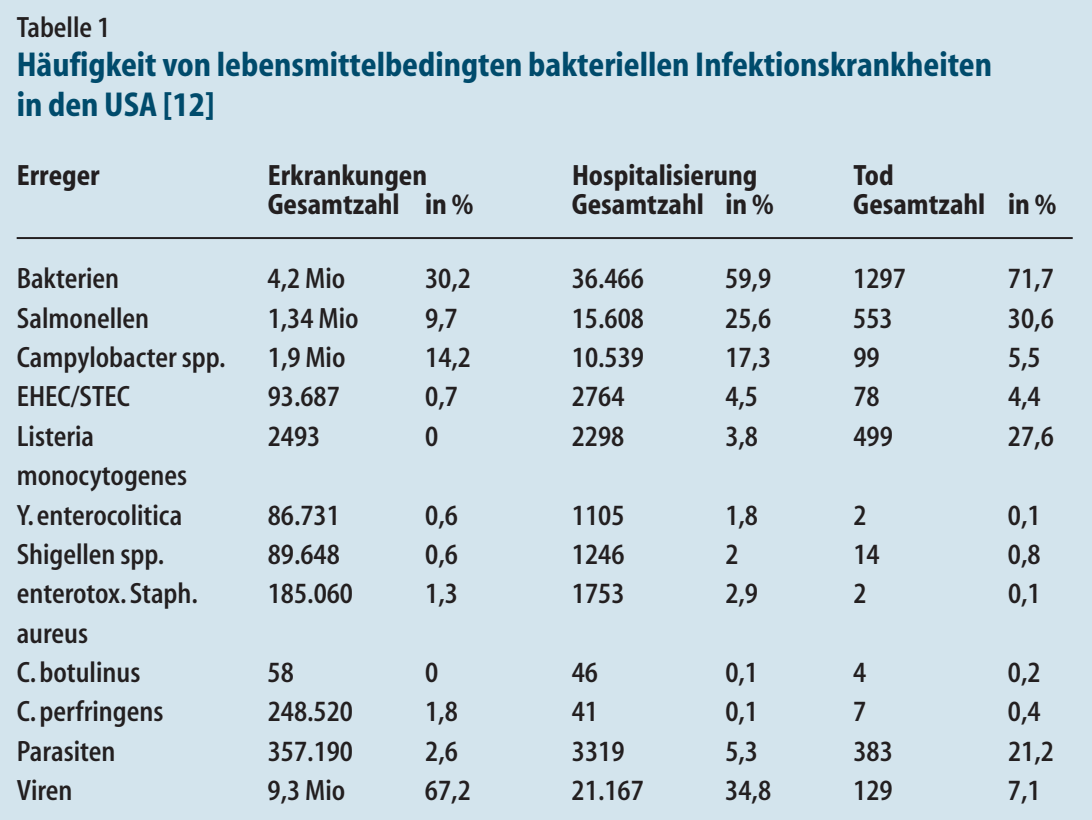


Tabelle 2

Erregervielfalt von bakteriell bedingten Erkrankungen durch Lebensmittel [vgl.4,5]

\begin{tabular}{lll} 
Erreger & Infektionsdosis & Inkub \\
\hline Bacillus anthracis & $10^{3}$ & $2-5$ Ta \\
& \\
& \\
Bacillus cereus & $>10^{6}$ (notwendig & $1-6 \mathrm{~h}$ \\
& $\begin{array}{l}\text { um eine genügende } \\
\text { Toxinemenge zu }\end{array}$
\end{tabular}

Brucella abortus, $\quad 10^{3}$

B. melitensis,

b. suis

Campylobacter
jejuni/coli

ca. 500

Clostridium

botulinum

Clostridium $\quad 10^{6}$

hängt von der gebildeten Toxinmenge $a b$

perfringens Typ A

Escherichia coli

10

EHEC (entero-

hämorrhagische) 2h-8 Tage gewöhnlich 12-48 h

$6-24 \mathrm{~h}$

mehrere Tage bis Wochen, oft $>30$ Tage

2-10 Tage, gewöhnlich 2-5 Tage

wässriger Durchfall (manchmal blutig), Bauchschmerzen, Fieber, selten das Guillain-Barrè-Syndrom

schwere neurologische Krankheitsbilder, Sehstörungen, Paralyse, Ateminsuffizienz

Durchfall, Bauchkrämpfe, Erbrechen und Fieber

Durchfall (oft blutig), Bauchkrämpfe (oft schwer), wenig oder kein Fieber, postinfektiös: HUS und TTP wässriger choleraähnlicher Durchfall, Bestimmung des Enterotoxins,

toxische)

\section{Escherichia coli}

$10^{5}$

EPEC (entero-

pathogene)

Escherichia coli

10

EIEC (entero-

invasive)
$12 \mathrm{~h}-6$ Tage

$6-48 \mathrm{~h}$

$12 \mathrm{~h}-6$ Tage gewöhnlich 2-4 Tage Bauchkrämpfe, Übelkeit, Erbrechen ohne Fieber

Isolierung der Erreger

Durchfall, Fieber, Bauchkrämpfe, lokale Ulzerationen

Durchfall (blutig), Fieber, Bauchkrämpfe
Anzüchtung der Keime, Differenzierung mit Hilfe von Virulenzmerkmalen zukünftig notwendig, Subdifferenzierung für epidemiologische Zwecke durch molekul. fingerprints biologischer Nachweis des Botulinus-Toxins, serologische Bestätigung

quantitative Anzüchtung der Keime, Bestimmung der Virulenzfaktoren (serologisch oder mit Hilfe von molekularbiologischer Methoden z. B.PCR) Bestimmung der Shiga-Toxinbildung als Leitmerkmal mit Hilfe serologischer und PCR gestützter Methoden, Isolierung und Typisierung der Erreger

Epidemiol. Besonderheiten

keine epidemiologische Bedeutung mehr in Europa

quantitative Anzüchtung

oft durch massive

Verunreinigungen klinische Bedeu-

tung davon abhängig, weist auf starke hygienische Mängel hin nur noch sporadisch, keine epideemiol. Bedeutung, Milch als Infektionsquelle, Laborinfektionen sehr weit verbreiteter Keim, besonders in Geflügelund Schweinebeständen

sehr selten durch verbesserte Lebensmittelhygiene , Erkrankung oft tödlich küchenhygienische Fehler bei Herstellung und Lagerung von

Speisen frühzeitige Diagnose notwendig zur rechtzeitigen

Unterbrechung der Kontaktketten, viele Erregerklone, breite Durchseuchung der Tierbestände als Reisedurchfall von Bedeutung, selten in Deutschland kaum noch epidemiol. Bedeutung Reisedurchfälle, nicht endemisch in Deutschland 
Tabelle 2

Erregervielfalt von bakteriell bedingten Erkrankungen durch Lebensmittel [vgl.4,5]

\begin{tabular}{|c|c|c|c|c|}
\hline Erreger & Infektionsdosis & Inkubationszeit & Krankheitssymptome & Nachweisverfahren \\
\hline $\begin{array}{l}\text { Listeria } \\
\text { monocytogenes a) }\end{array}$ & $10^{7}$ & 4 Tage-1 Woche & $\begin{array}{l}\text { Fieber, Meningitis, Sepsis neonatale } \\
\text { Blutvergiftung, selten Durchfall }\end{array}$ & $\begin{array}{l}\text { kultureller Erregernachweis und } \\
\text { PCR zur Erfassung der } \\
\text { Virulenzfaktoren für die } \\
\text { Differenzierung von } \\
\text { apathogenen Listerien }\end{array}$ \\
\hline $\begin{array}{l}\text { Mycobacterium } \\
\text { tuberculosis M. bovis }\end{array}$ & $<10$ & Wochen & $\begin{array}{l}\text { Lungentuberkulose, } \\
\text { ggf. andere Organe }\end{array}$ & $\begin{array}{l}\text { Erregernachweis, PCR } \\
\text { gestützter Nachweis }\end{array}$ \\
\hline $\begin{array}{l}\text { Salmonella enterica, } \\
\text { (verschiedene } \\
\text { Enteritis-Serovare) }\end{array}$ & $10^{2}-10^{6}$ & $\begin{array}{l}6 \mathrm{~h}-10 \text { Tage, } \\
\text { gewöhnlich 6-48 h }\end{array}$ & $\begin{array}{l}\text { Durchfall oft mit Fieber und Bauch- } \\
\text { krämpfen, } 5 \% \text { der Infizierten } \\
\text { (besonders Immungeschwächte) } \\
\text { entwickeln systemische Krankheitsbilde }\end{array}$ & $\begin{array}{l}\text { Anzüchtung der Keime, } \\
\text { Bestimmung der Serovare }\end{array}$ \\
\hline $\begin{array}{l}\text { Salmonella enterica, } \\
\text { Serovar Typhi, } \\
\text { Paratpyhi B }\end{array}$ & & $\begin{array}{l}\text { 3-60 Tage, } \\
\text { gewöhnlich } \\
\text { 7-14 Tage }\end{array}$ & $\begin{array}{l}\text { Fieber, Appetitlosigkeit, Unwohlsein, } \\
\text { Kopf- u. Muskelschmerzen, } \\
\text { grippeähnliche Symptomatik manch- } \\
\text { mal Durchfall oder Verstopfung }\end{array}$ & $\begin{array}{l}\text { Anzüchtung der Erreger, } \\
\text { Bestimmung der Antibiotika- } \\
\text { resistenz, Typisierung wichtig }\end{array}$ \\
\hline
\end{tabular}

Shigella spp. $\quad 10 \quad 12 \mathrm{~h}-6$ Tage

$\begin{array}{ll}\text { Staphylococcus } & \text { Toxinmenge } \\ \text { aureus } & \text { ist wichtig }\end{array}$

Vibrio cholerae $\quad 10^{3-10^{6}} \quad 1-5$ Tage

01,0139 , u.a.

Vibrio $\quad 10^{5}$

parahaemolyticus

Yersinia

$10^{4}$

enterocolitica

sonstige wie

$>10^{7}$

Enterococcus

faecalis/faecium,

Citrobacter freundii,

Klebsiella spp.,

Aeromonas hydrophila,

Plesiomonas shigelloides
Epidemiol. Besonderheiten

bei Immungeschwächten von Bedeutung

als lebensmittelbedingte Erkrankung in Deutschland praktisch ausgeschlossen verbreitet sich pandemisch

keine endemischen Typhusfälle, Import über Tourismus, beachte Antibiotika-

Mehrfachresistenz nicht mehr endemisch in Deutschland zunehmend weniger in Deutschland keine endemischen Gebiete, nur sehr selten über Tourismus importiert, oft ist Trinkwasser die Infektionsquelle sehr selten, meist aber über kontaminiertes Trinkwasser, oft auch Krebse u. a. Schalentiere in Deutschland ist nur der Serotyp 03 postinfektiös: rheumatische Gelenkbeschwerden fizierung, Kälteanreicherung, Nachweis des Virulenzplasmids, von Bedeutung Unterscheidung von apathogenen und pathogenen Yersinien Erregernachweis, schwierige Unterscheidung zwischen Kommensalen und Pathogeoft Hinweis auf unhygienische Verarbeitung Lebensmittel (biogene Amine) oder durch Pathogenitätsfaktoren wie Toxine, Adhäsine oder andere virulenzassoziierte Stoffwechselprodukte bedingt nen, Einsatz von molekularen

Methoden zur Charakterisierung des klinischen Potentials, Nachweis biogener Amine in Lebensmitteln 
te, durch Küchenfehler und andere hygienische Unzulänglichkeiten (z. B. Unterbrechung der Kühlkette, Vernachlässigung der Reinigungspflicht in der Küche etc.) kommt es u. U. zur rapiden Vermehrung dieser Erreger im Lebensmittel, in Folge dazu zur Infektion des Verbrauchers. Als Beispiel lässt sich das Salmonella Enteritidis-Problem zitieren: Das Hühnerei (im Ggs. zum Entenei) galt Jahrzehnte als sicher und für den Rohverzehr geeignet. Anfang der achtziger Jahre hat sich ein bestimmter Typ, der S. Enteritidis PT4 so an das Geflügel, insbesondere an das Hühnerei "angepasst”, dass er sich dort klinisch völlig unauffällig und auch nur in sehr geringen Keimkonzentrationen (ein mit 1-5 Keimen infiziertem Ei unter 500 nicht infizierten) festsetzen konnte [6]. Nach Verwendung derartig kontaminierter Eier traten $\mathrm{ab} 1985$ immer häufiger zum Teil sehr schwere Infektionen beim Menschen auf, was auch ein Ansteigen der Todesfolge nach Salmonellose mit sich brachte. Dieses Beispiel zeigt, dass ein Lebensmittel, das bisher als sicher galt, durch Veränderungen bestimmter Bakterieneigenschaften als hygienisches Problem in den Mittelpunkt gerückt werden kann, ohne dass am Verhalten der Hersteller oder der Lebenmitteltechnologie Schuldhaftigkeit festzustellen wäre. Der staatliche Gesundheitsund Verbraucherschutz der Bundesrepublik Deutschland und darüber hinaus in Europa nimmt die Sorge der Öffentlichkeit sehr ernst und hat zusätzliche weitreichende Maßnahmen zur Sicherung unserer Lebensmittel ergriffen $[7,8]$ wie z. B.:

D Schaffung einer EU-Zentrale für Lebensmittelsicherheit (europäische Lebensmittelagentur),

- Schaffung neuer EU-verbindlicher Richtlinien zur Bekämpfung von Zoonosen und anderen lebensmittelbedingten Krankheiten,

D Schaffung von EU-Forschungsschwerpunkten sowie von national geförderten Forschungsschwerpunkten zur Erfassung, Verhütung und Bekämpfung lebensmittelbedingter Infektionskrankheiten,

D Einrichtung von EU- und nationalen Referenzzentren zur Bekämpfung von lebensmittelbedingten Infektionskrankheiten (Tabelle 3).

\section{Allgemeine Aspekte der lebens- mittelbedingten Infektionskrankheiten}

Unabhängig von der Keimart ist für das Zustandekommen einer lebensmittelbedingten Infektionskrankheit häufig entscheidend, dass sich der Erreger im betreffenden Nahrungsmittel vermehren kann [4]. Die meisten Nahrungsmittel sind gute Nährmedien für alle betreffenden Erreger. Beim Botulismus und der Staphylokokken-Enterotoxikose führt diese Vermehrung zur Bildung entsprechender Toxine und damit zur Intoxikation (also der klassischen bakteriell bedingten Lebensmittelvergiftung). Bei einigen Keimgruppen ist erst eine sehr massive Vermehrung der Bakterien notwendig, wie für Clostridium perfringens oder Bacillus cereus, die nach Aufnahme ohne Kolonisation des Darmes durch ihre durchfallauslösenden Toxine wirken. Die meisten bakteriellen Erreger, die lediglich über die Lebensmittel zum Menschen transportiert werden, müssen aber zur Infektion und zur Auslösung der Krankheit erst im Darmtrakt kolonisieren und sich vermehren, wozu sie in der Regel geringere Infektionsdosen, aber ein breites Spektrum von Pathogenitätsfaktoren vorhalten müssen. (Tabelle 2, Abb. 1).

\section{Pathogenese und klinische Aspekte}

Erreger von Lebensmittelintoxikationen wie C. botulinum, enterotoxische Staphylokokken, Clostridium perfringens oder B. cereus bilden hochwirksame Toxine, die durchfallauslösende oder neurotoxische Wirkungen durch Interferenz mit dem zellulären metabolen Netzwerk ausüben, wie z. B die Fehlregulation der zellulären Immunabwehr durch die als Superantigene bezeichneten Staphylococcus aureus-Enterotoxine, die Blockierung der neuronalen Synapsen oder die ADP-Ribosylierung des Actins durch die Botulinus-Toxine oder die zerstörende Wirkung des C. perfringens-Toxins CPE für die tight junctions (zonula occludens). Bakterien, die das Lebensmittel nur als Transportvehikel nutzen, aber zur Auslösung der Erkrankung eine Kolonisierung und Vermehrung am Zielort benötigen, überwinden mit der Nahrung die Säureschranke des Magens, adhärieren - je nach Keimart - im Dünn- oder Dickdarm und lösen dort mit Hilfe ihre Pathogenitätsfaktoren (Invasine, Toxine, Moduline, Agressine) lokale Infektionsherde aus (Abb.1), von denen sie ausgehend auch zu systemischen Krankheitsverläufen "ausbrechen" können (besonders die Salmonellen). Dabei bedienen sich viele dieser Erreger (Salmonellen, Shigellen, Yersinien) einer sehr ökonomischen Einrichtung, in dem sie ihre Pathogenitätsfaktoren direkt in die Zielzelle einbringen können (durch das sog. Typ III-Sekretionssystem). Dadurch umgehen sie eine unnötige Überproduktion der Virulenzfaktoren, wie das z. B. für die Vibrio cholerae-Erreger der Fall ist, die eine mehr "zufällige" Aufnahme des Toxins in die Zielzellen erst durch dessen massive Freisetzung bedingen.

\section{"Die klinischen Hauptsymp- tome bakterieller Lebensmittel- vergiftungen sind Durchfall, Erbrechen, Schwindel, Bauch- krämpfe und Abdominal- schmerzen."}

Die Inkubationszeit für bakterielle Lebensmittelvergiftungen ist oft sehr kurz, sie kann aber erwartungsgemäß bei den richtigen Infektionsverläufen, wie z. B. bei Salmonellen und EHEC, mehrere Tage andauern. Als klinische Hauptsymptome sind Durchfall, Erbrechen, Schwindel, Bauchkrämpfe, Abdominalschmerzen (z.B. Shigellen) zu nennen,wobeisich postinfektiösschwerwiegende, oft lebensbedrohliche Syndrome entwickeln können, wie z.B. das hämolytisch-urämischeSyndrom(HUS) bei EHEC-Infektionen, das GuillainBarrè-Syndrom bei Campylobacter-Infektionen oder die Infekt-Arthritis nach Yersinia enterocolitica-Infektionen etc. Diese postinfektiösen Syndrome sind klinisch oft nur schwer zu beherrschen. Zur Therapie von Durchfällen gibt es vielfach konträre Ansichten. Als Faustregel ist festzuhalten, dass Durchfälle grundsätzlich nicht mit Antibiotika therapiert werden sollen, Ausnahmen dabei bleiben lediglich die Ruhrerkrankungen (Differentialdiagnose zu EHEC!) oder schwere typhoid verlaufende Salmonellosen (inkl. des Bauchtyphus). Gerade für die EHEC-bedingten Durchfälle ist die Antibiotikatherapie sogar kontraindiziert, da durch sie zwar das Wachstum 
Tabelle 3

Referenzeinrichtungen für die Überwachung und Erforschung lebensmittelbedingter bakterieller Infektionskrankheiten [21]

Bezeichnung

NRZ für Salmonellen und andere Enteritiserreger

Konsiliarlaboratorium für Listerien

Konsiliarlaboratorium für

Campylobacter und Aeromonas

Konsiliarlaboratorium für Clostridien

Konsiliarlaboratorium für

gastrointestinale Infektionen (Bakterien)

Konsiliarlaboratorium für

gastrointestinale Infektionen (Bakterien)

Nationales veterinärmedizinisches

Referenzlabor für Tuberkulose, (NRL-TUB)

Nationales veterinärmedizinisches

Referenzlabor für Brucellose (NRL-BRUC)

Aufgabe

Adresse

Diagnostik und Subdifferenzierung der Erregerisolate mit molekularen und klassischen Methoden für diagnostische und epidemiologische Zwecke, Erforschung der Virulenz und Resistenzentwicklung, Populationsanalyse der Erreger, Beratung und Unterstützung bei der Aufklärung von epidemischen Prozessen, zuständig für alle lebensmittelübertragenen bakteriellen Gastroenteritiserreger wie Salmonellen, Shigellen, E. coli, Campylobacter spp., Listeria monocytogenes, Vibrio cholerae, Vorhalten einer Stamm- und Referenzsammlung, Entwicklung und Anpassung neuer Methoden Diagnose, Subdifferenzierung und Pathogenitätsbestimmung von Listerien, Resistenzbestimmung

Diagnose und Subdifferenzierung von CampylobacterStämmen, Resistenzbestimmung

Toxinbestimmung und Differenzierung, Diagnose und Subdifferenzierung von Clostridien- Stämmen

Beratung bei Problemen bakteriell bedingter

Gastroenteritiden

Beratung bei Problemen bakteriell bedingter

Gastroenteritiden

Diagnose und Überwachung der Rindertuberkulose

Diagnose und Überwachung der Rindertuberkulose
Robert Koch-Institut, Bereich Wernigerode, Burgstr. 37, 38855 Wernigerode, Tel.: 03943-679237; Fax:03943-679207; E-Mail:tschaepeh@rki.de, Ansprechpartner:Prof.Dr. H. Tschäpe Hygiene Institut der Freien und Hansestadt Hamburg, Marckmannstr. 129a, 20539 Hamburg, Tel.:040-42837-201, Fax:040-42873-483, E-Mail:jochen.bockemuel@bags.hamburg.de, Ansprechpartner:Prof.Dr.J. Bockemühl

Institut für Medizinische Mikrobiologie und Hygiene, Fakultät Klin. Med. Mannheim der Univ. Heidelberg, Theodor-Kutzer-Ufer, 68167 Mannheim, Tel.: 0621-3832224, Fax:0621-383-3816, Ansprechpartner:Prof.Dr.H.Hof Institut für Med. Mikrobiologie und Hygiene, Klinikum der Univ. Freiburg, Hermann-Herder-Str. 11,79104 Freiburg, Tel.:0761-2036590/-6510, Fax:0761-2036562, e-mail:kistman@ukl.uni-freiburg.de, Ansprechpartner:Prof.Dr. M. Kist Thüringer Medizinal-, Lebensmittel- und Veterinäruntersuchungsamt, Abt. Medizinaluntersuchung Erfurt, FB Med. Mikrobiologie, Nordhäuser Str. 74, Haus 6, 99089 Erfurt,

Tel.:0361-740910, Fax:0361-7409113, Ansprechpartner:Dr. habil.H.-P.Schau Hygiene Institut der Freien und Hansestadt Hamburg, Marckmannstr. 129a, 20539 Hamburg, Tel.: 040-42837-201, Fax:040-42873-483, e-mail:jochen.bockemuel@bags.hamburg.de, Ansprechpartner: Prof. Dr.J. Bockemühl Institut für Med. Mikrobiologie u. Hygiene, Klinikum der Univ. Freiburg, Hermann-Herder-Str. 11,79104 Freiburg, Tel.: 0761-2036590/-6510, Fax:0761-2036562, e-mail:kistman@ukl.uni-freiburg.de, Ansprechpartner:Prof.Dr. M. Kist Bundesinstitut für gesundheitlichen Verbraucherschutz und Veterinärmedizin (BgVV) Jena, FG 41, Nauburger Str. 96a, 07743 Jena, Tel.:03641-804-240, Ansprechpartner: Prof. Dr. Schimmel Bundesinstitut für gesundheitlichen Verbraucherschutz und Veterinärmedizin (BgVV), FG 505, Diedersdorfer Weg 1, 12277 Berlin, Tel.: 030-84122053, Ansprechpartner:Dr.Staak 
Tabelle 3

Referenzeinrichtungen für die Überwachung und Erforschung lebensmittelbedingter bakterieller Infektionskrankheiten [21]

\begin{tabular}{|c|c|c|}
\hline Bezeichnung & Aufgabe & Adresse \\
\hline Nationales veterinärmedizinisches & Diagnose und Subdifferenzierung von Salmonellen aus & Bundesinstitut für gesundheitlichen \\
\hline $\begin{array}{l}\text { Referenzlabor für Salmonellen } \\
\text { (NRL-SALM) }\end{array}$ & Tierbeständen & $\begin{array}{l}\text { Verbraucherschutz und Veterinärmedizin } \\
\text { (BgVV), FG 501, Diedersdorfer Weg 1, } \\
12277 \text { Berlin, Tel.: 030-8412-2233, } \\
\text { Ansprechpartner: Dr. R. Helmuth }\end{array}$ \\
\hline $\begin{array}{l}\text { Nationales veterinärmedizinisches } \\
\text { Referenzlabor für E. coli (NRL-EC) }\end{array}$ & $\begin{array}{l}\text { Diagnose und Subdifferenzierung von E. coli, } \\
\text { insbesondere STEC, aus Tierbeständen }\end{array}$ & $\begin{array}{l}\text { Bundesinstitut für gesundheitlichen } \\
\text { Verbraucherschutz und Veterinärmedizin } \\
\text { (BgVV), FG 502, Jahnstr. 8, 06846 Dessau, } \\
\text { Tel.:030-8412-2201, } \\
\text { Ansprechpartner: Dr. Perlberg }\end{array}$ \\
\hline $\begin{array}{l}\text { Nationales veterinärmedizinisches } \\
\text { Referenzlabor für die Epidemiologie } \\
\text { der Zoonosen (NRL-E) }\end{array}$ & Erfassung von Daten über Zoonosen & $\begin{array}{l}\text { Bundesinstitut für gesundheitlichen } \\
\text { Verbraucherschutz und Veterinärmedizin } \\
\text { (BgVV), FG 501, Diedersdorfer Weg 1, } \\
12277 \text { Berlin, Tel.: 030-8412-2220, } \\
\text { Ansprechpartner: Dr. Hartung }\end{array}$ \\
\hline $\begin{array}{l}\text { Gemeinschaftliches Referenzlabor für } \\
\text { die Epidemiologie der Zoonose (CRL-E) }\end{array}$ & Erfassung von Daten über Zoonosen & $\begin{array}{l}\text { Bundesinstitut für gesundheitlichen } \\
\text { Verbraucherschutz und Veterinärmedizin } \\
\text { (BgVV), FB 5, Diedersdorfer Weg 1, } 12277 \text { Berlin, } \\
\text { Ansprechpartner: Frau Dr. Käsbohrer }\end{array}$ \\
\hline
\end{tabular}

der Keime zum Stillstand kommt, jedoch die Shiga-Toxine plötzlich massenhaft freigesetzt werden und so das Abwehrsystem des menschlichen Körpers einfach überfluten [9]. Auch sind das noch weit verbreitete Hausmittel "Kohle" und andere adsorbierende Substanzen zur Behandlung von Gastroenteritiden ungeeignet, da sie Wasser und damit auch die Erreger im Darmlumen binden. Eher sollten zusätzlich zu einer symptomatischen Behandlung die Verwendung von biologischen Präparaten, z.B. Mutaflor (ein E. coli-Präparat) oder Parenterol (ein Hefe-Präparat), in Betracht gezogen werden.

\section{Epidemiologie}

Lebensmittelbedingte Infektionskrankheiten gehören $\mathrm{zu}$ den häufigsten Krankheiten weltweit. In der Bundesrepublik beliefen sich z. B. die für 1998 gemeldeten Fälle von Enteritis infectiosa auf 212002 (darunter $46 \%$ Salmonellen) und für 1999 auf 195238 (darunter 43,5\% Salmonellen), was einer Inzidenz von ca. 340 pro $10^{5}$ der Bevölkerung entspricht. Mit einer 1997 durchgeführten Sentinelstudie zur Erfassung der Inzidenzen einiger wichtiger Gastroenteritiserreger $[10,11]$ ließ sich ermitteln, dass sich diese für Salmonellen auf $160 / 10^{5}$ (in den USA liegen diese Werte bei $26 / 10^{5}$ ), für
Shigatoxin-bildende E. coli-Bakterien auf $13 / 10^{5}$ und für Shigellen von $6 / 10^{5}$ beläuft (Abb. 2). Nach einer amerikanischen Studie [12] erkrankten in Amerika ca. 4 Mill. Menschen an bakteriellen lebensmittelbedingten Infektionskrankheiten, das sind ca. 30\% aller beobachteten, durch Lebensmittel übertragenen Erkrankungen (Tabelle 1). Jedoch sind ca. $60 \%$ aller schweren Krankheitsverläufe bakteriell bedingt, wobei Salmonellen mit etwa der Hälfte den Spitzenplatz einnehmen, ebenso sind $70 \%$ der letal verlaufenden lebensmittelbedingten Infektionskrankheiten durch Bakterien bedingt, wobei Salmonellen und Listerien zusammen fast $80 \%$ dieser Todesfälle ausmachen. Bemerkenswert dabei ist, dass gegenwärtig in den USA der hohe Anteil von Campylobacter spp. (ca.14\%) rückläufig zu sein scheint (von 1998 auf 1999 um 19\%) und dass gleichzeitig Salmonellen stark zunehmen $[13,14]$.

Als Infektionsquelle fungiert das kontaminierte Nahrungsmittel, dass entweder primär kontaminiert ist, z. B. Salmonella-haltiges Fleisch, Eier etc. oder aber sekundär bei der Vorbereitung, Zubereitung und Aufbewahrung der Lebensmittel in der Küche kontaminiert wird. Dabei ist zu vermerken, dass viele Lebensmittel erregerspezifische Besonderheiten zeigen, wie Salmonellen in eihaltigen Speisen, Listerien in Roh- milchkäse, C. botulinum bei Räucherfisch etc. Die lebensmittelbedingten Infektionskrankheiten treten vielfach als Gruppenerkrankungen auf [14],je nach dem, wie groß eine Essgemeinschaft ist.

\section{"Die primär infizierten Lebensmittel sind die wichtigste Infektionsquelle."}

Hier muss aber betont werden, dass ca. $85 \%$ aller lebensmittelbedingten Infektionen sporadischer Natur sind, also auf Einzelpersonen beschränkt bleiben, die durch die oft nur geringen Keimkonzentrationen oder durch einzelne küchenhygienische Fehler im Haushalt entstehen, so dass der Schwerpunkt der antiinfektiösen Maßnahmen auf eine diesbezügliche Aufklärung der Bevölkerung gelegtwerden muss.Da eine Kontamination der Lebensmittel durch symptomlose Ausscheider in Deutschland gegenwärtig sehr selten auftritt, sind die primär, zwar in der Regel, nur sehr gering infizierten Lebensmittel die wichtigste Infektionsquelle. Durch küchenhygienische Fehler reichern diese sich u. U. schnell an und können durch Übertragung auf andere Nahrungsmittel (z. B. das mit Salmonellen kontaminierte Auftauwasser, verunreinigte Arbeitsflächen in der Küche, Küchengeräte oder die 
Hände der Beschäftigten) ursprünglich hygienisch einwandfreie, oder durch Kochen, Garen, Braten keimfrei gemachte Lebensmittel verunreinigen (z. B. der bereits gefertigte Salat oder Pudding). Die Gründe für die gegenwärtig immer noch anhaltende Bedeutung lebensmittelbedingter Infektionskrankheiten sind (s. auch [15]):

D die Zunahme der Menschen, die außer Haus ihr Essen einnehmen,

D Veränderungen in der Abwehrlage der Bevölkerung (Zunahme der alten Bevölkerung etc.),

D Veränderungen im Ernährungs- bzw. Fehlernährungszustand sowie Reisen,

D die zunehmende Verwendung von tiefgefrorener Fertignahrung und Halbfertigprodukten,

D der Trend zum Verzehr von leicht Gegartem und Rohkost,

D die Konzentrierung von lebensmittelherstellenden Betrieben (riesige Chargen) mit langen Vertriebswegen und verlängertem MHD,

D die Globalisierung des internationalen Lebensmittelhandels (vgl. auch Tourismus),

D Veränderungen in der pathogenetischen Ausrüstung der Infektionserreger, aber auch Adaption an bestimmte ökologische Nischen.

\section{Bekämpfung und Prophylaxe}

Die beste Bekämpfung von lebensmittelbedingten Infektionskrankheiten ist die
Eliminierung der Erreger aus der Nahrungskette bzw. aus den Tierbeständen. Die Bemühungen zur Eradikation solcher Erreger aus den Tierbeständen waren in der Vergangenheit bei der Bekämpfung von Tuberkulose, Milzbrand und Brucellose sehr erfolgreich, sind aber für die gegenwärtigen Problemkeime Salmonellen, Campylobacter, EHEC und andere E. coli viel schwieriger und nur mit hohen ökonomischen Aufwendungen erreichbar. Diese Keimgruppen kommen ohne klinische Symptome, also ohne dass sie bemerkt werden könnten, in allen landwirtschaftlich genutzten Tierbeständen vor, sind aber auch in der Umgebung der Tierbestände in Ungeziefer, Ratten, anderen Umwelthabitaten (Wiese, Weide, Wasser) und dort über längere Zeit persistent. Eine mikrobiologische Erfassung von symptomlos ausscheidenden Tieren ist darüber hinaus schwierig, da die Keime intermittierend ausgeschieden werden und somit positive und negative Proben sich eher zufällig ergeben. Auch sind oft die betreffenden Keime nur in sehr geringen Konzentrationen vorhanden, so dass sie nur mit sehr speziellen und für Routinezwecke nicht sehr praktikablen Methoden nachzuweisen sind. Oft werden daher solche Tierbestände oder die von ihnen stammenden Rohprodukte für die Lebensmittelproduktion, z. B. Hühnerbestände, als salmonellafrei deklariert, die es in Wirklichkeit gar nicht sind. Deshalb kommt der Eigenkontrolle der lebensmittelherstellenden Betriebe eine

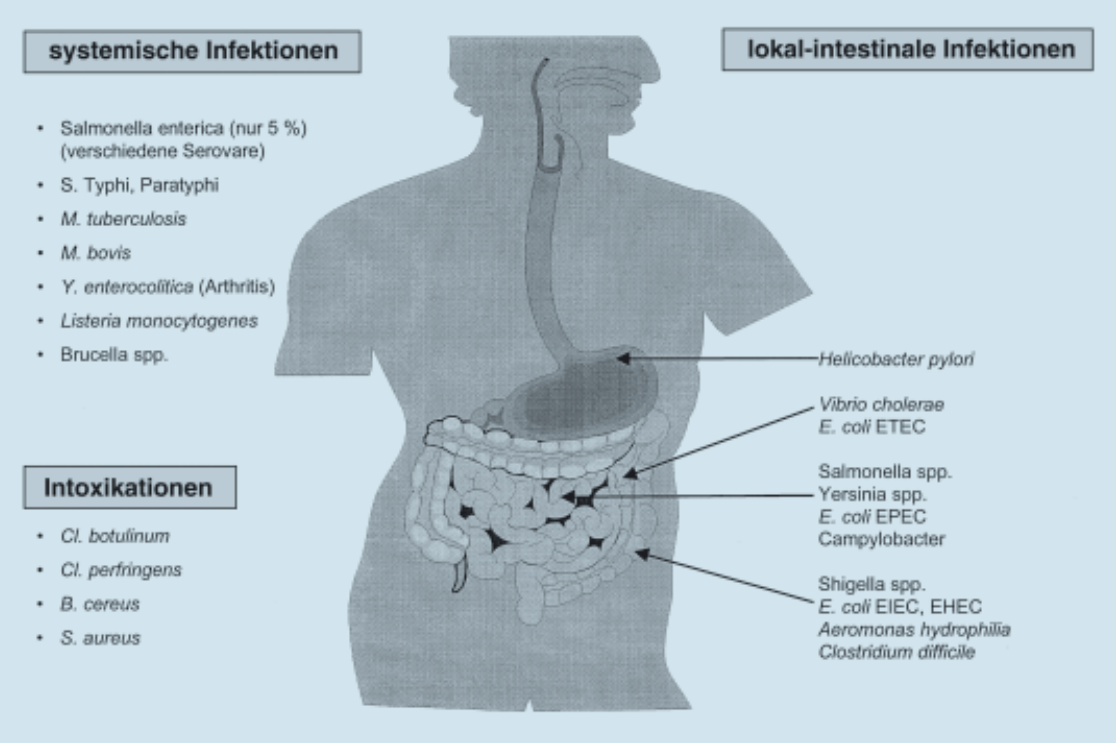

Abb. $1 \Delta$ Wirkorte der bakteriell bedingten, durch Lebensmittel übertragenen Infektionskrankheiten große Bedeutung im Verbraucherschutz $\mathrm{zu}$ (HACCP-Konzept), wobei insbesondere als wichtige prophylaktische Maßnahme bei der Herstellung von Lebensmitteln gilt, dass eine Vermehrung der Keime ausgeschlossen werden muss. Das betrifft vor allem die Lagerung und den Transport von Lebensmitteln und die besondere Beachtung bei der Herstellung von Rohprodukten (rohe Milch, rohes Fleisch, Hackfleisch, Speiseeis, Verwendung von rohen Eiern für Speisen).

\section{"Der Eigenkontrolle der lebensmittelherstellenden Betriebe kommt eine große Bedeutung im Verbraucher- schutz zu!"}

Da der Mensch in der Regel nicht als Infektionsquelle für die hier diskutierten Erregergruppen fungiert (Ausnahmen siehe Staph. aureus, teilweise auch EHEC) also eine Mensch-zu-MenschAnsteckung nur unter besonderen Umständen (Familie, Kindergarten, Kranken- und Altenpflege) eine Bedeutung haben, sind für die Wiederzulassung von Genesenen, aber noch in geringen Spuren ausscheidenden Patienten in Schulen und anderen Gemeinschaftseinrichtungen gegenwärtig weniger strikte Vorsichtsmaßnahmen üblich geworden, als das früher der Fall war [15]. Ausnahmen dazu sollten aber Kleinkinder sein (bis drei Jahre), insbesondere bei Vorliegen von EHEC-Infektionen, die bekanntlich nur eine sehr geringe Infektionsdosis aufweisen.

Zahlreiche prophylaktische Maßnahmen ergeben sich auch aus der Erfassung entsprechender Infektionskrankheiten und ihrer Surveillance, um Trends frühzeitig zu erkennen und geeignete Maßnahmen ergreifen zu können. Eine Meldepflicht für Erkrankungen und Sterbefälle durch lebensmittelbedingte Infektionskrankheiten, insbesondere Enteritis infectiosa, besteht gem. Bseuch G und ist in erweiterter und spezifizierter Form im neuen Infektionsschutzgesetz (IFSG) vorgesehen: es besteht danach eine Meldepflicht für Salmonella-Serovare, EHEC und Campylobacter sowie für das Auftreten von Gruppenerkrankungen nach Lebensmittelgenuss. 


\section{Erregerspezifische Besonder- heiten der lebensmittel- bedingten Infektionskrank- heiten}

Obwohl gegenwärtig alle bakteriellen Erreger von lebensmittelbedingten Krankheiten (vgl. Listeria monocytogenes, auch die klassischen Erreger wie Mycobacterium bovis, Bacillus anthracis, Brucella melitensis, B. abortus, B. suis etc.) besonders Gastroenteritiden verursachen (Abb. 1), weisen sie eine breite biologische und pathogenetische Vielfalt mit vielen klinischen und epidemiologischen Besonderheiten auf (Tabelle 2).

\section{Lebensmittelbedingte Intoxikationen bakteriellen Ursprungs}

\section{Clostridium botulinum}

Lebensmittelintoxikationen durch das Botulinus-Toxin haben dank hygienisch verlässlicher Lebensmitteltechnologien, besonders durch genügendes Erhitzen und hohe Kochsalzkonzentrationen, weitgehend an Bedeutung verloren. Da allein die durch das Bakterium produzierten Toxine die neurologischen Krankheitssymptome auslösen und diese durch Temperaturen über $60^{\circ} \mathrm{C}$ inaktiviert sowie durch ausreichende Salzkonzentrationen oder $\mathrm{pH}$-Absenkung in ihrer Ausbildung gehindert werden, sind trotz des ubiquitären Vorkommens dieser Keime ernstzunehmende lebensmittelbedingte Ausbrüche in den letzten 25 Jahren nicht mehr zu verzeichnen gewesen. Da sie sich ubiquitär in Bodenhabitaten verbreitet haben, ist im Prozess der Lebensmittelherstellung jederzeit die Möglichkeit gegeben, dass entsprechende C. botulinum-Sporen in die Fertigware geraten und bei entsprechendem Nachlassen der Vorsicht wieder zeitweise an Bedeutung gewinnen können (vgl. jüngstes Beispiel bei einem Thüringer Wursthersteller). Beim Botulismus ist therapeutisch eine schnelle Serumabgabe von Bedeutung, so dass durch die neutralisierenden Antikörper die Toxinwirkung vor ihrer Bindung an der motorischen Endplatte aufgehoben werden kann. Da das Botulinum-Toxin in sehr niedrigen Konzentrationen wirkt, sind schon geringe Spuren ohne merkbare Qualitätsveränderung der Lebensmittel klinisch wirksam.

\section{Staphylococcus aureus}

Enterotoxinbildende Stämme sind unter S. aureus weit verbreitet (ca. 40\%). Solche Stämme sind für etwa $1 \%$ der lebensmittelbedingten Infektionskrankheiten verantwortlich (Tabelle 1). Die Toxine werden vor der Nahrungsaufnahme gebildet (allerdings immer über Temperaturen von $15^{\circ} \mathrm{C}$ ), so dass sie präformiert im Lebensmittel vorliegen. Eine kurze Inkubationszeit von ca. 1-4 h ist typisch für die Staphylokokken-Enterotoxikose, was sich auch anamnestisch verwenden lässt. Die S. aureus-Toxine (es sind zahlreiche verschiedene Toxine bekannt und als A, B, C1, C2, C3, E, D, G, $\mathrm{H}$, I, und $\mathrm{F}$ bezeichnet) interferieren als sog. Superantigene mit der zellulären Immunantwort, was zu einem Überschießen der freigesetzten Zytokine führt und sekundär Reaktionen wie Erbrechen, Fieber, Kreislaufkollaps, Histaminausschüttung usw. hervorruft. Eine Behandlung der Staphylokokken-Enterotoxikose ist nur symptomatisch möglich, da die relativ hohe Wirksamkeit der Enterotoxine schon bei geringen Spuren klinische Symptome bedingen kann, so dass man die betroffenen Lebensmittel auch als hygienisch einwandfrei einstuft. Als Infektionsquelle für diese Krankheit fungiert in der Regel der Mensch, der zu 30 bis 50\% mit Staph. aureus-Stämmen (Nase) kolonisiert ist. Auch können kleine eitrige Wunden an den Fingern als Kontaminationsquelle fungieren. Durch Unachtsamkeiten und hygienische Mängel geraten die enterotoxischen Stämme in die Lebensmittel.

\section{Clostridium perfringens und Bacillus cereus}

Im Unterschied zu C. botulinum und den enterotoxinbildenden S. aureus sind Lebensmittelvergiftungen durch $\mathrm{C}$. perfringens und $\mathrm{B}$. cereus nur nach einer massiven Vermehrung dieser Keime möglich (weit über $10^{6} / \mathrm{g}$ ). Ihre Bedeutung als Lebensmittelvergifter ist daher rückläufig und weist immer auf extreme hygienische Mängel bei der Lebensmittelherstellung hin (Tabelle 2). Strikte Einhaltung der Hygienestandards verhindert solche Lebensmittelvergiftungen.

\section{Lebensmittelbedingte bakterielle Infektionen}

\section{Salmonellen}

Salmonellen, die mit der Nahrung aufgenommen werden (vgl. die Bedeutung dieser Keime für das Trinkwasser), überstehen die Magenpassage leicht, weil sie oft durch die protein- und fetthaltige Nahrung gegen die Magensäure geschützt werden. Erst im Darmtrakt bilden sie ihre Pathogenitätsfaktoren aus und führen zu lokalen Erkrankungen des unteren Dünndarms (Abb. 1). Die Salmonellen sind trotz zahlreicher antiepidemischer und antiinfektiöser Strategien in den letzten 20 Jahren ein ständiges Problem geblieben [9]. Obwohl die Salmonellose in Deutschland seit 1992 stark rückläufig ist (von $1992 \mathrm{zu}$ heute um die Hälfte), bleiben sie noch mit knapp 100000 die am häufigsten registrierte lebensmittelbedingte Erkran-

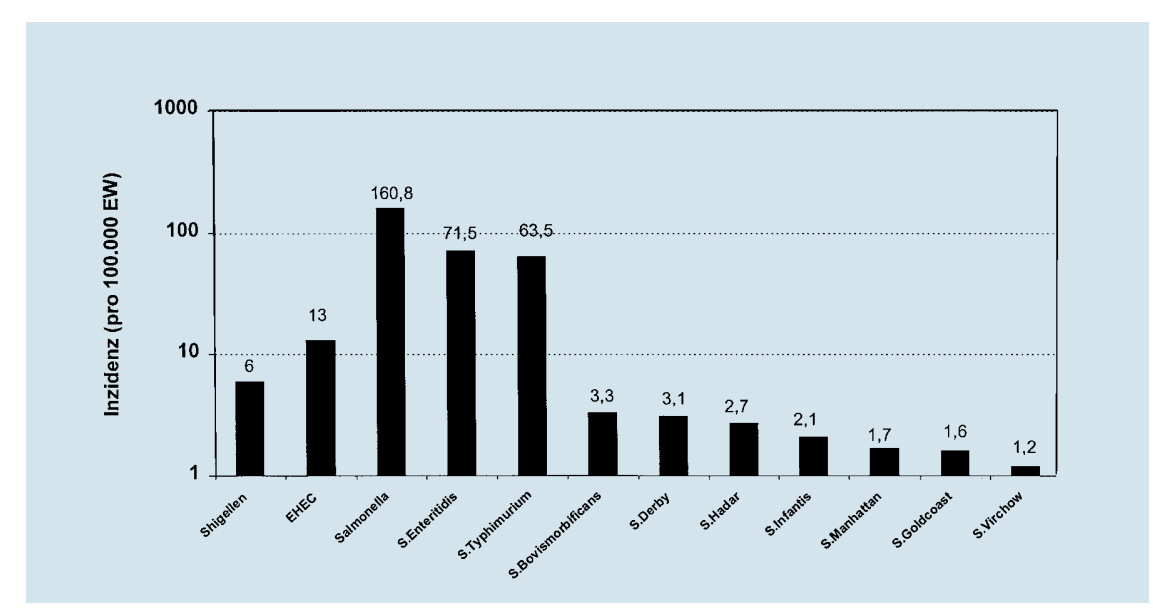

Abb. $2 \triangle$ Inzidenzen von Salmonellen, Shigellen und EHEC Bakterien in Deutschland gemäß einer Sentinelstudie $1997[10,11]$ 
kung. Dabei steht S. Enteritidis mit ca. $45 \%$ an der Spitze (Abb. 2). Salmonellen verfügen über ein breites Spektrum von Virulenzfaktoren, den sog. Effektorproteinen, die von zwei Typ III-Sekretionssystemen direkt und in sehr geringen Konzentrationen in den Zielzellen appliziert werden. Diese Virulenzproteine stören verschiedene zelluläre Stoffwechselvorgänge und führen zu Fehlleistungen des Organismus, z. B. zur Fehlregulation des Wasser- und Elektrolythaushalts oder zur Aufnahme und Vermehrung der Salmonellen in die Zielzellen (z. B. Makrophagen).

\section{"Epidemiologisch gesehen tritt beim Menschen nur eine sehr geringe Zahl von Salmonella-Epidemietypen auf."}

Trotz der großen Fülle von Serovaren, Varianten und Stämmen, die Salmonellen in der Natur erkennen lassen, tritt beim Menschen epidemiologisch gesehen nur eine sehr geringe Zahl von Epidemietypen auf, die sich offenbar für den epidemischen Prozess besonders qualifiziert haben [6]. So war z. B. der Epidemietyp S. Enteritidis PT4 über Jahre hinweg in der "Salmonella-Landschaft" unbekannt, avancierte dann jedoch zwischen 1984 und 1992 zum dominierenden Epidemietyp. Seit 1992 befindet er sich wieder auf dem Rückzug, 1999 hat er das Niveau von 1987 erreicht $[2,16]$. Obwohl er mit ca. 55\% immer noch der häufigste Epidemiestamm ist, hat sich inzwischen ein weiterer Salmonella-Epidemietyp auf den Vormarsch gemacht, der zum Serovar Typhimurium gehört und als DT104 bezeichnet wird. Dieser Stamm besitzt eine breite Antibiotikamehrfachresistenz, was besondere Komplikationen dann impliziert, wenn der betroffene Patient zusätzlich zum Durchfall ein typhoides Krankheitsbild entwickelt. Was solche dominanten Salmonella-Epidemiestämme charakterisiert, ob sie bestimmte Qualifizierungen (epidemische Virulenz) aufweisen, an denen sie zu erkennen und von anderen Nichtepidemietypen zu unterscheiden sind oder warum sie wieder aus der "Szene" verschwinden, sind einige der vielen Fragen, die zu beantworten bisher noch offen bleibt.
Besondere epidemiologische Bedeutung haben in den letzten paar Jahren Salmonella-Ausbrüche und Einzelfälle gefunden, die auf den Genuss von Sprossen zurückzuführen waren. Dies zeigt, dass sich Salmonellen nicht nur über Fleisch- und Eiprodukte ausbreiten können, sondern auch über pflanzliche Nahrungsmittel. Wahrscheinlich haften die Salmonellen an den Samen der Pflanze oder haben das Gießwasser kontaminiert. Als heute in Deutschland nicht mehr endemische Salmonellose gilt der Typhus [17]. S. Typhi-Bakterien werden heute nur noch im Ausland (Tourismus) aber dort stets über Lebensmittel (oft auch Trinkwasser) erworben. Weil der Typhus stets durch Antibiotika therapiert wird, kommt der Antibiotikaresistenz und ihrer Überwachung eine große Bedeutung zu [17].

\section{Shigella spp.}

Die verschiedenen Shigalla-Spezies (sonnei, flexneri, boydii, dysenteriae) sind in Deutschland seit einigen Jahren nicht mehr endemisch, sondern treten nur als Importe (Lebensmittel, Tourismus) auf. Durch ihre niedrige Infektionsdosis werden Shigellen meistens von Mensch zu Mensch verbreitet und weniger über Lebensmittel. Eine besondere Bedeutung für die Verbreitung von Shigellen als Lebensmittelvergifter nehmen Milchprodukte, z. B. Käse, ein. Da die Shigellose in der Regel mit Antibiotika behandelt wird, kommt der Antibiotikaresistenz und ihrer Überwachung eine große Bedeutung zu.

\section{Campylobacter spp.}

Zu den häufigsten Durchfallerregern in Europa und Amerika gehören Campylobacter jejuni/coli, die jedoch meistens nur leichte bis mittelschwere Durchfälle (vgl. aber das Guillain-Barrè Syndrom) und weniger spektakuläre Ausbrüche hervorrufen [14]. Campylobacter ssp. sind besonders in Geflügel- und Schweinebeständen weit verbreitet (gegenwärtig ist eine Häufung von Infektionen über kontaminierte Rohmilch zu verzeichnen) und lassen sich auch bei gesunden Menschen vielfach nachweisen. Über die Pathogenese ist bisher nicht viel bekannt, so ist $z$. B. noch strittig, ob sie lediglich eine lokale toxische Reaktion oder eine lokale Invasion (wie Shigel- len) hervorrufen [18]. Auch die verschiedene Bedeutung besonders hinsichtlich ihrer Virulenz qualifizierter Stämme ist unbekannt. Durch ihre Fähigkeit, das Genom umzubauen (genetic rearrangement), lassen sich Stämme epidemiologisch nur über kurze Zeiträume verfolgen. Seit den letzten drei Jahren wird z. B. in den USA dank verbesserter Hygiene in den Geflügelbeständen ein starker Rückgang der Campylobacter-Infektionen registriert $[13,14]$. Da Campylobacter spp. als thermophile Keime sich oberhalb von $30^{\circ} \mathrm{C}$ nicht vermehren und eine besondere Empfindlichkeit gegenüber Umwelteinflüssen aufweisen, ist ihre Vermehrung im Lebensmittel in der Regel nicht gegeben.

\section{Vibrio spp.}

Infektionen durch Vibrio cholerae und parahaemolyticus sind in Deutschland sehr selten und nur im Ausland erworben. Sie spielen nur bei importierten Lebensmitteln (meist Fisch und andere Meerestiere) eine Rolle. Vibrionen sind eigentlich sog. Wasserkeime, die besonders durch verschmutztes Trinkwasser verbreitet werden. Viele bakterielle Erreger werden auch über Trinkwasser verbreitet, z. B. EHEC, Shigellen, S. Typhi. Epidemiologisch gesehen gibt es keinen Unterschied zwischen durch Trinkwasser und Lebensmittel übertragenen Infektionen.

\section{Yersinia spp.}

Weniger als 1\% lebensmittelbedingter bakterieller Infektionskrankheiten sind in Deutschland durch Yersinia enterocolitica bedingt (vgl. auch Tabelle 1), die überwiegend der Serogruppe O3, weniger häufig $\mathrm{O}_{9}$ und $\mathrm{O}_{5,27}$ angehören und durch die Anwesenheit des yersiniatypischen Virulenzplasmids gekennzeichnet sind. Yersiniaarten sind weit verbreitet, jedoch gehören die meisten von ihnen apathogenen Formen an. Humanpathogene Y. enterocolita-Stämme haben ihren Ursprung in Schweinebeständen und vermehren sich insbesondere durch einen längeren Aufenthalt im Kühlschrank (Kälteanreicherung) im betroffenen Lebensmittel zur relevanten Infektionsdosis. Yersinia pseudotuberculosis kommt in Deutschland und Westeuropa selten vor, kann aber in Osteuropa und Asien (Russland) eine epidemiologische 
Bedeutung einnehmen. In den USA gibt es zusätzlich virulente Stämme, die zu einem besonderen Biovar (Biovar1B) gehören. Infektionen mit humanpathogenen Yersinien können im Anschluss an die Darminfektion in eine Infektarthritis übergehen, die sich über Wochen bis Monate erstrecken kann. Hier liegt in der Mehrzahl der Fälle eine genetische Disposition der Patienten zu Grunde (HLA B27).

\section{Enterohämorrhagische E. coli (EHEC)}

EHEC-Bakterien unterscheiden sich konventionell nicht von den normalen E. coli-Bakterien der gesunden Darmflora und können nur mit Hilfe einer Reihe molekularbiologischer Methoden durch den Nachweis ihrer Virulenzfaktoren differentialdiagnostisch charakterisiert werden [9]. EHEC-Bakterien sind gegenwärtig weltweit epidemisch im Vormarsch und sind evolutionär offenbar erst in jüngster Zeit entstanden (horizontaler Gentransfer). Als Reservoir der EHEC-Bakterien gelten insbesondere Rinderbestände, so dass besonders Rindfleisch- und Milchprodukte (Rohwürste, Rohmilch, Rohmilchkäse, ggf. auch Sprossen, unpasteurisierte Säfte) als Infektionsquelle dienen. Durch ihre niedrige Infektionsdosis (wie Shigellen) können auch sehr geringe Konzentrationen an EHEC-Bakterien Krankheitssymptome auslösen. Da postinfektiös durch die Wirkung ihrer Toxine lebensbedrohliche Syndrome besonders bei Kleinkindern entstehen können (wie das HUS), muss rechtzeitig durch einen gesicherten Nachweis der Erreger eine weitere Ansteckung verhindert wie auch frühzeitige Intervention zur Verhinderung des HUS geführt werden.

Die aus einer Sentinelstudie [16] hervorgegangene Einschätzung zur Häufigkeit von EHEC-Infektionen in Deutschland zeigte (Abb. 2), dass sie offenbar mit 13/105 nur 1:10 so oft wie Salmonellen vorkommen (vgl. auch Tabelle 1). Da EHEC-Infektionen besonders im Kleinkindesalter schwerwiegende klinische Bilder erzeugen, sollten besonders diese vor dem Genuss von Rohprodukten geschützt werden [1]. Die Überwachung der EHEC-Infektionen und ihrer Erreger ist dringend notwendig. Dabei ist es besonders wichtig, Virulenzausrüstung und Serotypzugehörigkeit der Erreger festzustellen.
Enterotoxische E. coli (ETEC), säuglingspathogene E. coli ( EPEC) und enteroinvasive E. coli (EIEC)

Ähnlich wie die EHEC-Bakterien, die sich nur durch den Erwerb von Pathogenitätsdeterminanten von den E. coliStämmen der normalen Darmflora unterscheiden, sind auch andere Pathovare wie ETEC, EPEC und EIEC nur durch die Charakterisierung der verschiedenen Virulenzausrüstungen zu diagnostizieren. Die ETEC kommen in Deutschland und den nördlichen Industriestaaten selten, meist unter $1 \%$ vor [1]. Sie verursachen die als sog. Reisediarrhoe bezeichnete, oft lästige Begleiterscheinung einer Reise in subtropische Länder. Auch die enteroinvasiven E. coli (EIEC) sind als Erreger der Reisediarrhoe besonders auf dem Balkan und in Südostasien häufig. Die in früheren Zeiten durch Hospitalismus verbreiteten enteropathogenen E. coli-Bakterien (EPEC) spielen gegenwärtig in Deutschland keine epidemiologische Rolle. Alleiniges Erregerreservoir dieser drei E. coli Pathotypen ist der Mensch.

\section{Listeria monocytogenes}

Durch lebensmittelbedingte Infektionen durch L. monocytogenes sind zwar selten, scheinen aber in den letzten Jahren zuzunehmen [14]. Sie treten in Rohmilchprodukten (Käse, Camembert), Rohwürsten, etc. auf. Da es sich bei ihnen um Erreger meist schwerer systemischer Krankheitsbilder (sehr selten Durchfall) handelt, werden Fälle von Listeriosen (Fieber, Kopfschmerzen, grippeähnliche Symptome) deutlicher wahrgenommen als es bei den Durchfällen der Fall ist. Listerien sind weit verbreitet, allerdings betrifft das vor allem die apathogenen Listerien, z. B. Listeria innocua, die durch ihre hohe "Durchseuchung" zu einer Wirtsimmunität gegen L. monocytogenes führt. Daher werden Infektionen mit L. monocytogenes nur bei Risikogruppen wie Schwangeren (grippeähnliche Symptome) und Neugeborenen (Sepsis und Meningitis), alten Menschen (Sepsis) und Immungeschwächten (Sepsis) registriert. Als Krankheitserreger des Menschen ist gegenwärtig vor allem der Serovar $4 \mathrm{~b}$ von L. monocytogenes verbreitet. Eine Antibiotikatherapie ist angezeigt.

\section{Lebensmittelbedingte Infektions- krankheiten ohne gegenwärtige seuchenhygienische Bedeutung}

Infektionsherde mit Brucella melitensis, B. abortus, B. suis, Mycobacterium bovis (tuberculosis), B. anthracis oder Francisella tularensis sind in Deutschland erloschen, und eine Übertragung über einheimische Lebensmittel ist daher auszuschließen. Durch die Globalisierung des Handels bleibt das Thema noch aktuell.

\section{Sonstige Erreger von Lebensmittel- infektionen mit unklarer Bedeutung}

Ein großer Anteil der ätiologisch nicht aufzuklärenden Lebensmittelerkrankungen (ca. 65\%) könnten durch eine Reihe von Erregertypen bedingt sein, die zu den ätiologisch und pathogenetisch bisher nicht klar definierten Erregergruppen wie Citrobacter freundii, Edwardsiella torda, Plesiomonas shigelloides, Aeromonas hydrophila, Enterococcus faecalis, Pseudomonas aeruginosa und anderen sog. fakultativ-pathogenen Darmbakterien gehören. Ihre pathogenetische Ausrüstung ist unvollständig bekannt, oft treten diese Spezies auch in Folge von hygienischen Mängeln in der Lebensmittelherstellung auf. Solche Keimgruppen können auch durch hohe Keimkonzentrationen zu sog. unspezifischen Lebensmittelvergiftungen insbesondere durch Bildung biogener Amine führen [5]. Manche dieser Erreger lassen sich öfters bei Immungeschwächten als Erreger nachweisen, so Enterococcus faecalis und E. faecium bei hospitalisierten Personen. Über das Vorkommen solcher Keime wird immer wieder berichtet. Es fehlen molekularepidemiologische Studien, die bestimmte Zusammenhänge herstellen können.

\section{Schlussfolgerungen für die Praxis}

Zwar besteht im Rahmen des BSeuchG eine Meldepflicht für Durchfallerkrankungen (Enteritis infectiosa) einschließlich mikrobiell bedingter Lebensmittelvergiftungen, jedoch erfolgt diese als Krankheitsbild und nicht erregerbezogen. So beliefen sich z. B. die für 1999 gemeldeten Fälle von Enteritis infectiosa auf 195238, doch welche Erregergruppen mit ihren sehr verschiedenen pathogenetischen und epidemiologischen Eigen- 
schaften beteiligt sind, bleibt dabei unbekannt. Auch sind die rückläufigen Meldezahlen (um ca. 10\% von 1998 auf 1999, siehe [3]) wissenschaftlich und gesundheitspolitisch nur schwer auszuwerten, weil diese entweder durch erfolgreiche Interventionsmaßnahmen, durch verbesserte Kenntnisse der Bevölkerung, durch eine erfolgreiche Reduktion der Erregerlast in den Tierbeständen oder aber durch das vielfach zu beobachtende epidemiologische Auf und Ab bestimmter Erregergruppen [19] bedingt sein können. Im ab 2001 verbindlichen Infektionsschutzgesetz (IfSG) ist der Verdacht auf und die Erkrankung an einer mikrobiell bedingten Lebensmittelvergiftung oder einer akuten infektiösen Gastroenteritis meldepflichtig, sobald zwei oder mehr zusammenhängende Erkrankungen vorliegen.

Zwar konnte in einer Sentinelstudie des NRZ für Salmonellen und andere Enteritiserreger $[10,11]$ in einem niedersächsisch-thüringischen Einzugsgebiet die Gesamtzahl an gemeldeten Salmonellen etwa bestätigt werden, was zeigt, dass das Meldeverhalten in der Bundesrepublik gar nicht so schlecht ist, doch bleibt die getrennte Erfassung der verschiedenen Salmonella-Serovare, der pathogenen E. coli-Serovare, insbesondere der EHEC, der Campylobacter spp., Yersinia ssp. etc. für eine qualifizierte und weiterführende epidemiologische Schlussfolgerung insbesondere im europäischen Vergleich unbedingt erforderlich. Das soll mit dem neuen Gesetz zur Verhütung und Bekämpfung von Infektionen beim Menschen, dem Infektionsschutzgesetz (IfSG), für die Bundesrepublik verbindlich geregelt werden. Damit sind dann nicht nur gezielte Interventionsmaßnahmen, sondern auch eine bessere internationale Vergleichbarkeit $\mathrm{zu}$ erreichen. Auch die notwendige und geforderte Einbindung der Bundesrepublik in die EUweit (z. B. EnterNet) bzw. WHO-weit (Global Salm-Surv) verknüpften epidemiologischen Netzwerke, wie es die EUDirektive [8] vorsieht, erfordert eine den modernen Ansprüchen angepasste Meldeform für Infektionskrankheiten. Entsprechend des für 2001 verbindlichen IfSG ist der Verdacht auf und die Erkrankung an einer mikrobiell bedingten Lebensmittelvergiftung oder einer akuten infektiösen Gastroenteritis meldepflichtig, wenn zwei oder mehr zusammenhängende Erkrankungen vorliegen. Dabei muss jedoch davon ausgegangen werden, dass $85 \%$ der lebensmittelbedingten bakteriellen Infektionskrankheiten sporadischer Natur sind, also oft durch individuelle küchenhygienische Fehler in den Haushalten der Bevölkerung entstehen, und dass nur die spektakulären Ausbrüche in der Öffentlichkeit eine große Presse finden. Daher muss neben der strengen Durchsetzung des HACCP Konzeptes in der Lebensmittelindustrie die Aufklärung der Bevölkerung hinsichtlich einer verbesserten Küchenhygiene erfolgen, wozu die Medien, aber auch populäre und griffig-einleuchtende Aufklärungsschriften verbreitet werden müssen [z.B. 20]. Darüber hinaus ist eine laborgestützte Surveillance dieser Erregergruppen insbesondere auch durch die Hilfe der entsprechenden Nationalen Referenzlaboratorien (Tabelle 3) für eine rechtzeitige Erkennung von Ausbrüchen und Trends unerlässlich, wie sie ja erfolgreich bereits in anderen europäischen Ländern wie den Niederlanden, England und Wales, Schweden, Dänemark etc. übliche Praxis ist.

Daher ergeben sich folgende Schlussfolgerungen:

D Meldepflicht der lebensmittelübertragenen Bakterien nach Serovaren und Erregertypen wie im IfSG vorgesehen, was eine komplexe Diagnostik im niedergelassenen Bereich voraussetzt,

D laborgestützte Surveillance von lebensmittelübertragenen bakteriellen Infektionserregern wie SalmonellaSerovaren, E. coli-Pathovaren, Shigella spp., Listeria monocytogenes, Campylobacter spp.u.a.,

D Mitarbeit in den europaweiten Netzwerken,

D Verfolgung von Infektketten, insbesondere bei internationalen Ausbrüchen,

- Reduktion der Erregerlast in den Tierbeständen (Stallhygiene, Durchimpfung, Schlachthygiene), Durchsetzung von Regeln der Grundhygiene und des HACCP-Konzept in der Lebensmittelindustrie.

Danksagung. Herrn Prof. Dr. Jochen Bockemühl danke ich für seine kritische Durchsicht des Manuskripts und seine anregenden Kommentare.

\section{Literatur}

1. Bockemühl J (1996) Salmonellen, EHEC-Bakterien und andere Lebensmittelinfektionen. In: Freie Hansestadt Hamburg (Hrsg) Ansteckend. Berichte und Informationen zum Thema Infektionskrankheiten. Edition Temmen, Bremen, S 32-39

2. Robert Koch-Institut (1999) Zur Situation bei wichtigen Infektionskrankheiten im Jahr 1998. Teil 1: Darminfektionen (Gastroenteritiden). Epid Bull 15/99:99-106

3. Robert Koch-Institut (2000) Wochenstatistik ausgewählter Infektionskrankheiten.: Epid Bull 7/2000:58

4. Jay JM (1992) Modern food Microbiology, 4th edn. Chapman and Hall, New York London

5. Doyle MP (1989) Foodborne bacterial pathogens. M. Dekker, Inc. New York Basel

6. Kühn H, Tschäpe H (1996) Salmonellosen des Menschen - ätiologische und epidemiologische Aspekte. RKI-Schrift 3/95, Münchner Medizin-Verlag

7. Europäische Gemeinschaft Zoonose-Richtlinie. EU Directive 92/117/EWG

8. Europäische Gemeinschaft. Epidemiologische Netzwerke. EU Directive 2119/09/EC. Amtsblatt der europ. Gemeinschaften - Rechtsvorschriften L268, Jahrgang 41:1-7

9. Karch H, Schmidt H, Schwarzkopf A (1996) Enterohämorrhagische $E$. coli (EHEC). Die gelben Hefte. Immunbiol Informationen 36:125-135

10. Gericke B, Claus H, Voigt M, Tschäpe H, Rasch G, Holler H, Wagner H (1999) Die epidemiologische Situation der Salmonellose in Deutschland 1997. Bundesgesundheitsbl 42:196-205

11. Prager R, Reissbrodt R, Holler H et al. (1998) Isolierung und Charakterisierung von Shigatoxin-produzierenden E. coli-Stämmen aus Stuhlproben Ergebnisse einer Sentinel-Studie. Bundesgesundheitsbl 41 [Sonderheft Okt. 98]:6-13

12. Mead PS, Slutsker L, Dietz V et al. (1999) Foodrelated illness and death in the United States. Emerg Infect Dis K5:607-625

13. Centers for Disease Control and Prevention (2000) Weekly Report. Preliminary FoodNet data on the incidence of foodborne illnesses - selected sites, United States 1999. MMWR 49:201-205

14. Olsen SJ, MacKinnon LC, Goulding JS, Bean NH, Slutsker L (2000) Surveillance for foodbornedisease outbreaks - United States, 1993-1997.In: CDC-Surveillance Summaries. MMWR 49 SS:1-64

15. Merkblatt für Ärzte des RKI und des BgVV (1996) Empfehlungen für die Wiederzulassung in Schulen und sonstigen Gemeinschaftseinrichtungen. Deutscher Ärzteverlag, Köln

16. Tschäpe $H$, Liesegang A, Gericke $B$, Prager R, Rabsch W, Helmuth R (1999) The up and down of salmonella enterica serovar enteritidis in Germany. In: Saeed AM (ed) S. enterica serovar enteritidis in humans and animals. lowa State Univ. Press./Ames Purdue Univ Press:51-61

17. Tschäpe H (1999) Ansteckungsgefahr durch Typhus-Bakterien bei Reisen ins Ausland. Immun Impfen 2:60-66

18. Ketley JIM (1997) Pathogenesis of enteric infections by Campylobacter. Microbiology 143:5-21

19. Tschäpe H (1996) Ursachen des Auf und Ab der Salmonellose des Menschen. Bundesgesundheitsbl 39:260-263

20. Reckitt, z Colman z Deutschland AG (1999) Erkennen und Vermeiden. Geselle Salmonelle und andere Übeltäter. Hamburg

21. Robert Koch-Institut (1997) Nationale Referenzzentren und Konsiliarlaboratorien:Verzeichnis der Laboratorien und Leistungsübersicht. Eigenverlag, Berlin 